\title{
Our Voices Matter: Using Lived Experience to Promote Equity in Problem Gambling Prevention
}

\author{
Victor Ortiz ${ }^{1} \cdot$ Rachael Cain $^{1} \cdot$ Scott W. Formica ${ }^{2} \cdot$ Rebecca Bishop $^{3} \cdot$ Haner Hernández ${ }^{3} \cdot$ Lorena Lama $^{1}$ \\ Published online: 28 May 2021 \\ (C) This is a U.S. government work and not under copyright protection in the U.S.; foreign copyright protection may apply 2021
}

\begin{abstract}
Purpose of Review The field of problem gambling has been historically disconnected from the community experience of gambling and people of color, leading to a lack of integration of those with lived experience into programming. The aim of this article is to describe community-centered efforts to prevent and mitigate harm from problem gambling in Massachusetts — including a pilot program, the Massachusetts Ambassador Project, which is grounded within public health and lived experience frameworks.

Recent Findings To engage Massachusetts communities in problem gambling prevention, planning processes were conducted to develop culturally appropriate prevention strategies. One of the recurrent themes was the desire of men in the substance misuse recovery community to share their knowledge with others, specifically, men of color who experience racism and health disparities. This finding informed the development of the Ambassador Project, a novel, peer-based, community-centered, and culturally responsive approach for men of color who have a history of substance misuse to engage other men of color in problem gambling prevention.

Two organizations pilot tested the project and reached 4388 individuals. The pilot led to several findings in the design and implementation of related projects. Lessons are shared in three categories: structure, support, and implementation.

Summary This article demonstrates an innovative approach to connect the field of problem gambling prevention to the community experience, using a public health and social justice lens. Others in the field should acknowledge the disconnect between problem gambling and the lived experience of those disproportionately impacted by creating opportunities for community voice to be at the center of programming.
\end{abstract}

Keywords Problem gambling $\cdot$ Lived experience $\cdot$ Equity $\cdot$ Public health $\cdot$ Gambling recovery $\cdot$ Gambling prevention

\section{Introduction}

\section{Background}

Problem gambling ${ }^{1}$ is a public health and social justice issue that impacts individuals, families, and communities. Research

\footnotetext{
${ }^{1}$ There are many terms to describe an individual who is experiencing an adverse and stressful association with gambling. In this article, we utilize the term "problem gambling" as an addiction disorder marked by compulsive behavior with the potential to result in personal, interpersonal, and societal harm [4]. Although there are various prevalence estimates of problem gambling nationally, attention is lacking on those who are disproportionality impacted by it and on the factors which elevate risks and harms to those populations.

This article is part of the Topical Collection on Gambling

Victor Ortiz

Victor.ortiz@mass.gov

1 Massachusetts Department of Public Health, Boston, MA, USA

2 Social Science Research and Evaluation, Inc., Burlington, MA, USA

3 EDC, Waltham, MA, USA
}

shows that communities of color and individuals with mental health disorders and substance use disorders are disproportionately impacted by gambling $[1 \bullet \bullet, 2 \bullet, 3]$. Such impacts lead to inequities that may contribute to negative social, economic, and health outcomes.

The field of problem gambling prevention has been historically disconnected from the community experience of gambling and people of color [4]. Such a divide has led to a lack of community engagement and integration of those with lived experiencedefined here as people who have direct and indirect experience with recovery from or exposure to gambling - into research and programming. These individuals may also be referred to as experts by experience. Voices of such populations matter - without their engagement, there can be exacerbated health disparities and a lack of focus on the social determinants of health (described below). As opportunities to gamble grow, it is imperative that researchers and practitioners give critical thought to how best to engage communities in order to prevent and mitigate risk, harm, and impacts of gambling and related issues.

Limited research in the field of gambling has begun to include the voices of those with lived experience to 
understand their unique perspectives on the issue and to solicit their assistance in designing more effective interventions. In one study, researchers interviewed individuals with experience of gambling-related problems and found that the use of responsible gambling narratives led to feelings of stigmatization and negative stereotypes while having a limited impact on helpseeking behaviors [5•]. In another study, the researchers interviewed people who experienced gambling harms to contrast their perspective with that of the gaming industry and related implications for prevention interventions [6]. Other research has also demonstrated the critical value of integrating lived experience in the development and implementation of interventions. For example, Marshall and colleagues conducted a systematic review of the ways in which the substance misuse harm reduction movement has engaged a population - people who inject drugs - into the development, testing, and interpretation of programs and services to increase their relevance and effectiveness [7•]. Additionally, Watling and colleagues conducted a rapid review of research using lived experience of suicidality in intervention development, concluding that limited studies on the topic exist and that increasing such expertise in intervention development would be valuable [8].

The aim of this article is to describe community-centered efforts to prevent and mitigate harm from problem gambling in the Commonwealth of Massachusetts-including an innovative pilot program, the Massachusetts Ambassador Project, which is grounded within a lived experience framework. The project is led by the Massachusetts Department of Public Health (MDPH) Office of Problem Gambling Services (OPGS). OPGS aims to address the disconnect between those disproportionately impacted by gambling and the field through innovative programs centered on data and community voice from those most impacted.

The Massachusetts Ambassador Project was designed to acknowledge the comorbidity between gambling, substance addiction, and mental health disorders, the need for holistic and comprehensive prevention strategies that emphasize the health of the whole community, and the disproportionate impact of problem gambling on historically marginalized and oppressed populations. Emerging evidence highlights the multiple pathways through which gambling, substance use, and other behavioral health issues manifest within different segments of the population [3,9]. This body of work reiterates the co-occurrence of these behaviors and the need for customized approaches based on ascriptive characteristics such as race, sex, and ethnicity. Peer-based interventions grounded in a lived experience framework provide a way to leverage shared risk and protective factors in meaningful and relevant ways between the senders and receivers of information. All aspects of the Ambassador Project development and design were informed by the principles of health equity, racial equity, and a focus on local community control, the value of wisdombased practice, and lived experience.
The sections that follow describe OPGS' public health approach to problem gambling, community engagement methods, intervention development based on lived experience, and lessons learned. We will also outline how OPGS uses an equity-focused public health approach to work alongside communities to center lived experience in their problem gambling prevention work.

\section{A Problem Gambling Public Health Approach Grounded in Social Justice}

A public health approach grounded in social justice is critical to responding to the lived experience of those impacted by problem gambling. A public health approach to problem gambling prevention and treatment is proactive and encourages a shift from a narrow focus on just individual gamblers to a broader consideration of the social setting; the social, cultural, and economic factors that influence the spread and patterns of a disorder $[10 \bullet, 11]$. Public health approaches are driven by data and acknowledge - through the socio-ecological model, in particular - the intersection and impact of individual, interpersonal, community, and systems levels on health outcomes [12].

Problem gambling is governed by a complex set of interrelating factors [13]. Figure 1 illustrates the application of the socio-ecological model to problem gambling, demonstrating its multifaceted and interconnected nature. By directly applying this model to problem gambling, policymakers, prevention practitioners, and communities are better able to visualize and address gambling on the individual, interpersonal, community, and systems levels.

Public health also focuses on the social determinants of health. The social determinants of health are "conditions in the places where people live, learn, work, and play that affect a wide range of health and quality of life risks and outcomes," such as employment and education [14]. Approximately 80$90 \%$ of health outcomes are linked to the social determinants of health [15]. The social determinants of health affect those impacted by problem gambling. For example, individuals who have access to strong social supports are more likely to be protected from impacts related to problem gambling [16]. Such impacts are exacerbated for populations who experience inequities (e.g., not having strong social support while also lacking housing intensifies the impact of the former).

In Massachusetts, OPGS emphasized the application of a socio-ecological model to problem gambling prevention that actively considered social determinants of health within an overarching social justice framework. Central to this framework were the concepts of equitable access to resources and services, participation of individuals with lived experience, respect, humility, and beneficence. In contrast to the traditional focus of gambling interventions - treatment for an individual - this approach concentrated on the holistic, 
Fig. 1 Application of problem gambling to the socio-ecological model

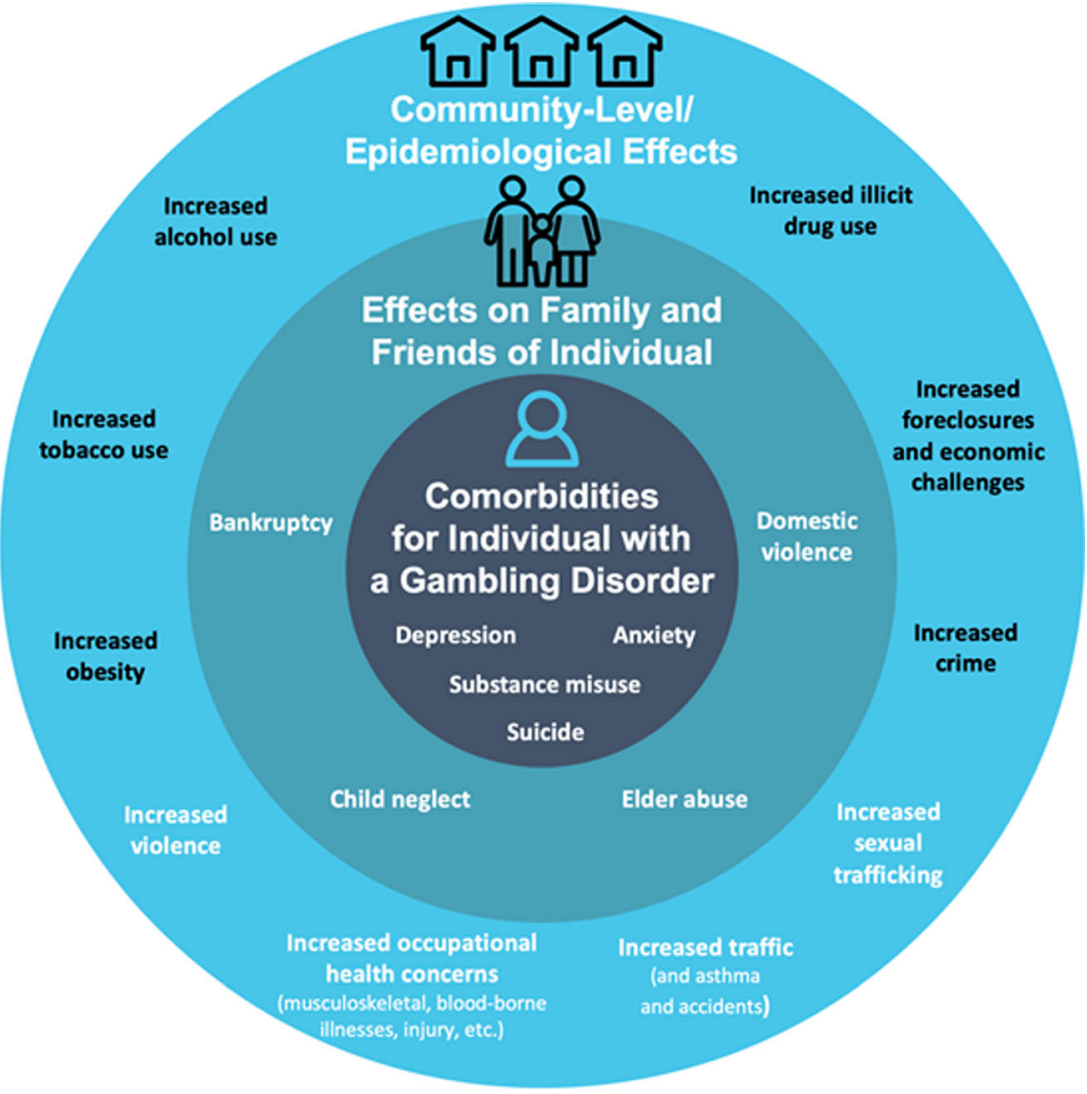

multidimensional nature of problem gambling and innovative ways to address the issue, centered around lived experience and community engagement of those most impacted.

\section{Leading With Equity: Community Engagement Practices}

MDPH promotes the health and well-being of all residents by ensuring access to high-quality public health and healthcare services, and by focusing on prevention, wellness, and health equity [17]. Within MDPH, OPGS leads a public health response to problem gambling, guided by evidence and community voice, while ensuring cultural intelligence and humility. Critical to the work of MDPH and OPGS are data and the social determinants of health, with a vision of eradicating health disparities.

OPGS recognized the need to establish a comprehensive public health response to mitigate problem gambling and its related harms due to increased opportunities to gamble in the state. OPGS' public health approach, rooted in the voices of those with lived experience, is novel in the field. This approach moves beyond traditional deficit-based problem and responsible gambling programs. Traditional methods tend to create interventions in a silo and then bring the intervention to the community. This jeopardizes cultural appropriateness and effectiveness. Organizations cannot expect interventions to be appropriate for the community if those with lived experience are not actively engaged in the planning process [6].

Community engagement is central to any public health process or intervention. It involves engaging those affected by an issue in a way that allows the impacted population(s) to learn about the risks they face, participate in acceptable response actions [18], and share their knowledge.

To engage Massachusetts communities in problem gambling prevention, OPGS initiated three planning processes across the state, beginning in 2017 [16, 19]. Two priority populations were identified, one of which was men of color who have a history of substance misuse [19]. The planning process was conducted to identify and/or develop culturally appropriate prevention strategies grounded in health equity principles.

A guiding principle of the planning process was the belief that community members, particularly those with lived experience, should inform the identification and development of resources, supports, and messages that would resonate with individuals at elevated risk of problem gambling. Each regional planning process included the following: (1) outreach to community members and service providers; (2) convening of local stakeholders concerned about the potential impact of expanded gambling in their region; (3) identification of community assets, local resources, and gaps in services; and (4) conducting key stakeholder interviews and focus groups. 
Two hundred and sixty-five Massachusetts residents were engaged across all three regional planning processes to inform the planning and design of prevention initiatives by OPGS and its partners. Key stakeholders were community members, including those with lived experience, who had expertise in community issues and how to address them, and who both lived and worked in the region(s). Similarly, focus group participants across the assessments included priority populations such as men in recovery from a substance use disorder [19]. Some of those engaged did not necessarily have experience with gambling-related harms - their knowledge as an informed community member or member of a community disproportionately impacted by gambling provided a meaningful contribution in OPGS' public health planning approach. In acknowledgement of the co-occurring nature of gambling and other behavioral health issues, many stakeholders from the well-established substance misuse recovery community in Massachusetts participated in these groups and interviews. Although these individuals may not have had direct lived experience with problem gambling, these individuals were able to leverage their experience with other related issues, to advance the discussion and identify opportunities for aligning work and breaking down artificial funding and programming silos. This ensured people with both direct and indirect lived experience were meaningfully involved in the planning processes by design [6].

All participants in the planning process described their concern for certain populations that may be at high risk for problem gambling, including men, people of color, and people with a history of substance misuse or comorbid mental health conditions [19]. Collection and analysis of qualitative data informed the development of prevention messaging that incorporated local context and concerns for the identified priority populations. The community context explored racial demographics, immigrant populations, and populations that experience social and economic hardship. Community organizations that play a role in addressing these issues or other community needs were also included.

\section{Equity in Action: Development of Prevention Strategies}

Massachusetts prevention initiatives for problem gambling were largely informed by the regional planning process, placing the lived experience of community members at the center of program development. During the regional planning process, one of the recurrent themes was the desire of men in the substance misuse recovery community to share their knowledge and experience with others, including the well-documented relationship between substance misuse and gambling [20, 21]. This finding informed the development of the Massachusetts Ambassador Project, a novel, peer-based, community-centered, and culturally responsive approach for men of color who have a history of substance misuse to engage other men of color in their communities in problem gambling prevention discussions through individual, group, and community-level interactions.

\section{The Massachusetts Ambassador Project: Project Design}

In 2018, OPGS contracted with two recovery-oriented organizations to pilot test the Ambassador Project and to participate in a formative evaluation. These two organizations were also provided with intensive training and technical assistance from the Massachusetts Center of Excellence on Problem Gambling Prevention (MCOE PGP), a state-supported training and technical assistance center housed within a non-profit organization.

The goals of the project were to (a) prevent or reduce problem gambling among individuals, priority populations, and communities with elevated or disproportionate levels of risk and (b) develop and foster the awareness, skills, and behaviors needed to protect against developing problems with gambling. In developing the specific parameters for the project, OPGS drew extensively from the literature on peer support interventions [22], health and behavioral health education and outreach $[23,24]$, and brief interventions $[25,26]$. The use of peers to deliver prevention, health promotion, and recovery support services is commonplace across a wide variety of fields, including substance misuse prevention, HIV/AIDS prevention, and violence prevention. As described by Valente and Pumpuang [27], peer providers, including but not limited to those with direct lived experience with the health or behavioral health issue being addressed, tend to be more adept at using appropriate language and expressions, are often intrinsically motivated to help others with shared experience, and can be perceived as being a more credible source of information than non-peers. Other investigators have noted that peer providers are uniquely and experientially qualified to draw on their lived experience to establish or reinforce norms, leverage resources within local settings, and reach members of historically difficult-to-engage populations $[27,28]$.

Theoretically grounded in the socio-ecological model, the MA Ambassador Project included a comprehensive set of objectives and corresponding activities at the individual, interpersonal, organizational, and community levels. The focus at the individual level was on training, empowering, and supporting two to three Ambassadors in each recovery organization as catalysts of change. While the core of their knowledge is experiential and based on lived experience, the literature suggests that peer providers should also receive structured training consistent with other paid staff in areas such as ethics, coaching skills, relationship building, public speaking, and peer education [7•]. Upon hire, MCOE PGP provided each Ambassador with 15 hours of training on problem gambling prevention, the relationship between substance use disorder and problem gambling, building health and racial equity, and 
engaging peers in group and individual conversations. This training was supplemented by ongoing opportunities for training, technical assistance, and professional development. Ambassadors were encouraged and supported in pursuing certification as recovery coaches and were invited to participate in monthly community of practice sessions with other Ambassadors for peer sharing and support. Within their host organization, Ambassadors also received weekly supervision and support from a dedicated project supervisor.

The objective at the interpersonal level was for Ambassadors to actively engage, involve, and empower individuals disproportionately affected by or at risk for problem gambling. Following training, Ambassadors were expected to use lived experience to raise awareness of problem gambling, emphasize the relationship between substance misuse and problem gambling, provide resources, and support behavioral change through individual and group interactions. Individual interactions tended to be time-limited and based on capitalizing upon natural opportunities to engage individuals in discussion (e.g., street outreach). Group interactions were more structured in nature and based on requests to provide presentations at partner organizations such as acute treatment facilities, institutional corrections facilities, and faith-based settings.

At the organizational level, Ambassadors established relationships with community-based organizations that prioritize men of color and/or allies in recovery. The objective was to effect systems change by encouraging these organizations to institutionalize problem gambling prevention within their services, enhance their capacity to support problem gambling prevention, and meet the needs of individuals at elevated risk on the shared risk factor of gambling and substance addiction.

At the community level, Ambassadors were expected to increase community awareness of problem gambling and provide education about susceptibility to problem gambling - including recognition of environmental exposure to gambling and the connection between problem gambling and other health and behavioral health issues. To this end, Ambassadors engaged with the broader community through the distribution of OPGS informational materials and resources at community events.

\section{The Massachusetts Ambassador Project: Pilot Period Outcomes}

The 12-month pilot implementation period, which lasted from July 2018 to June 2019, was externally evaluated [29]. Emphasis was placed on understanding the extent to which Ambassadors were able to successfully engage with the community, the feasibility of the project, and the identification of needed adjustments to the activities in advance of moving the project to scale. The primary evaluation inputs were an online monthly reporting form completed by the project supervisor at each recovery-oriented organization, multiple site visits with the supervisor and Ambassadors at each project site, and process discussions with representatives from OPGS and MCOE PGP.

Over the course of the pilot period, Ambassadors reached a total of 4388 individuals through individual outreach, group sessions and presentations, and material dissemination. Throughout this, Ambassadors reached communities of color - critical to engaging a population disproportionately impacted by gambling. For example, $43 \%$ of the individuals who participated in the group sessions and presentations identified as a person of color (1326/3067). In addition to their direct work with individuals and groups, Ambassadors engaged and developed relationships with 36 different community organizations.

Initial anecdotal reports from supervisors, Ambassadors, OPGS, and MCOE PGP suggest the project enhanced the capacity of Ambassadors to act as catalysts of change in the community, successfully engaged individuals and organizations in lived experience discussions about problem gambling, and was well received by individuals and the community as a whole. One Ambassador stated, "They like my group because I am honest with them and they can sense that... they are more engaged because they feel more familiar with me and know what to expect." The project is currently being replicated in four settings and plans are underway to conduct a more robust process and outcome evaluation.

\section{Lessons Learned: Practical Take-Aways From the Experience}

The initial pilot implementation period of the Ambassador Project resulted in the identification of several key findings that may help guide others in the design and implementation of related projects grounded in equity within a lived experience framework. These lessons fall into the three broad categories of structure, support, and implementation.

Structure refers to characteristics of the host organization within a community-based setting and is inclusive of readiness, capacity, philosophical alignment, and systems of management and supervision. Support refers to external resources and processes that are intended to enhance operation of the project and facilitate its functioning. Implementation refers to direct actions that can be taken to increase the extent to which the program is relevant, appropriate, effective, and accepted by members of priority populations and by the community as a whole. See Fig. 2 for a visual overview.

\section{Structure}

Consideration should be given to which organization or entity will host the program and its readiness and capacity. The program could be hosted within a wide variety of settings (e.g., municipal health department, multi-service center, harm 
Fig. 2 Lessons learned from the Massachusetts Ambassador Project pilot
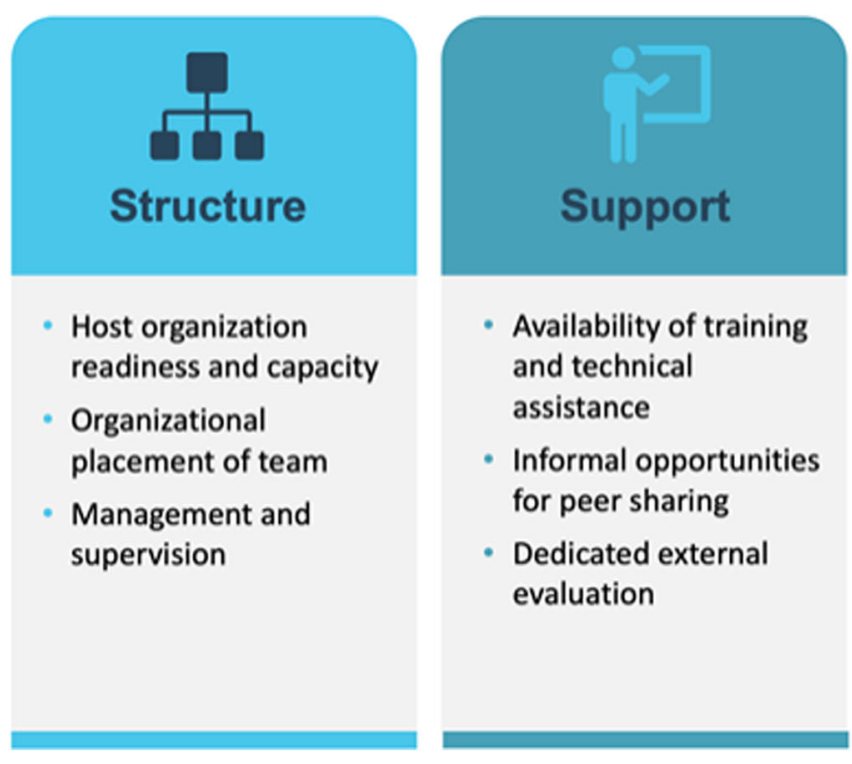

- Availability of training and technical assistance

- Informal opportunities for peer sharing

- Dedicated external evaluation

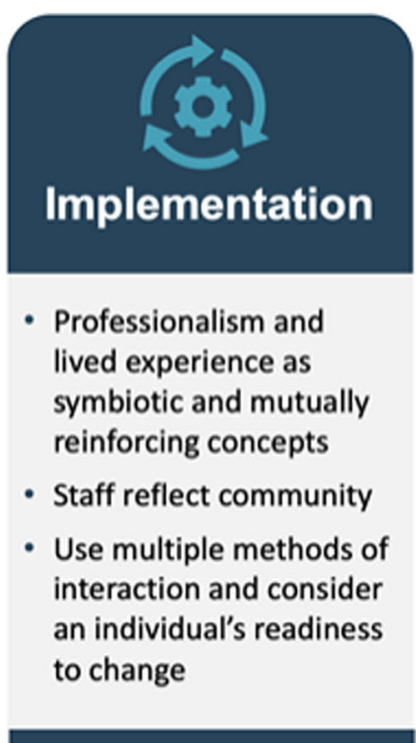

reduction agency) provided that the site is capable of accommodating an outreach worker, peer coach, or recovery coach with lived experience. In Massachusetts, the Ambassador Project was housed within the state's existing network of recovery support centers. These centers are sanctuaries anchored in the heart of the community that offer culturally and linguistically appropriate education, activities, and information that support all paths to health and wellness. The Ambassador Project aligned with the mission, philosophy, and approach that already existed within these settings and was able to capitalize on established systems of management and supervision.

Once a setting has been identified, decisions need to be made concerning whether outreach workers will be volunteers, receive a stipend, or be hourly or salaried employees and whether they will be allotted physical space within the organization and associated resources (e.g., laptop). For the Ambassador Project, Ambassadors were paid a salary and given physical office space and associated resources. The frequency, purpose, and content of management and supervision processes should also be determined beforehand and articulated, including a set of well-defined expectations.

\section{Support}

Ambassadors had access to external training, technical assistance, and evaluation support. From the outset of the project, the importance of formal and ongoing professional development related to problem gambling prevention, ethics, recovery coaching, and health and racial equity were emphasized as a supplement to lived experience.

In addition to individual customized technical assistance and group or conference-based training opportunities, Ambassadors were also routinely convened in informal communities of practice. In contrast to more formal supports, the communities of practice provided opportunities for peer sharing and the exchange of information and best practices within the cohort of Ambassadors across multiple implementing sites. A key finding among training, technical assistance, and evaluation providers was the need to actively involve Ambassadors in the planning of activities, foster an environment marked by open communication, trust, and mutual respect, be flexible to changing plans in response to new information, and to approach the project from a place of cultural humility.

\section{Implementation}

From an implementation perspective, a fundamental lesson learned was the need to simultaneously promote professionalism and lived experience as symbiotic and mutually reinforcing concepts. As suggested by the literature [27], Ambassadors reported that they were viewed as being more credible and were better received when they were able to share their personal experiences and speak from a place of lived experience. On the other hand, Ambassadors often spoke of the need to be seen as members of the professional workforce and expressed a desire for branding materials, business cards, and other markers - particularly when interacting with representatives from community-based agencies. While not all outreach workers will be in a position to interact with both community members and agency representatives, this finding illustrates the need to provide these individuals with the tools to easily adapt to and seamlessly flow between a multitude of settings with different audiences.

Ambassadors highlighted the importance of understanding the community's needs and resources, having clarity of purpose, and understanding the manner in which individuals prefer to access and receive information. Ambassadors also felt that the 
project needed to be staffed by individuals who reflect the cultural, linguistic, and gender composition of the community. Having Ambassadors who are fluent in the dominant languages and dialects within the community and being able to provide gender-specific services, as needed, were both provided as specific examples.

Knowledge of the community, including its history, geography, needs, and resources, was also viewed as being an essential asset. Ambassadors spoke to the need to understand the manner in which community members access and utilize available services and knowing which messages might resonate with different individuals and segments of the community. For example, in large urban settings which tend to be highly neighborhood-based, understanding prevailing microculture norms, places where people congregate, and dominant verbal communication channels can enhance the relevance and salience of outreach activities and services.

Attention to setting and preference was also identified as being a consideration at the individual level. Ambassadors stressed the importance of not relying on a single type or method of interaction. For example, some individuals prefer receiving written information, some prefer group work, and others prefer one-to-one discussions. In relation to the latter, Ambassadors reported that one-on-one discussions provide an opportunity for some individuals to share personal struggles and concerns about themselves or family members that may not emerge during larger group sessions and presentations.

Specific to the population of focus in this project, men of color with a history of substance misuse, Ambassadors commented on capacity and readiness to change and on specific attitudes and norms within segments of the recovery community. Individuals who are in the early stages of recovery from substance use disorder may have low capacity and readiness to simultaneously consider problem gambling behaviors. Ambassadors need to be aware of these issues and understand how to appropriately engage this population.

Similarly, Ambassadors need to be aware of the prevailing culture and norms within the institutions in which they are providing services. Some recovery-oriented organizations emphasize multiple pathways to recovery-sometimes called a "no wrong door" philosophy. Other recovery-oriented organizations emphasize a single or preferred path to recovery (e.g., 12 -step models). Just as there should be philosophical alignment between the project and the host organization, Ambassadors emphasized that there should be some level of philosophical alignment between the project and the institutions in which outreach is conducted.

\section{Conclusion}

This article demonstrates MDPH OPGS' innovative approach to connect the field of problem gambling prevention to the community experience, using a public health and social justice lens. Intensive and purposive community engagement drives the creation of OPGS' evidence-informed problem gambling prevention activities and places lived experience at the center of all of the office's work.

Others considering how to begin or enhance problem gambling work should acknowledge the disconnect between problem gambling prevention and the lived experience of those disproportionately impacted-specifically, those that have been historically marginalized and oppressed and face the greatest level of inequities - by creating opportunities for community voice to be at the center of program development and implementation. The lessons learned regarding structure, support, and implementation described above can be a guide for such activities. Evidence-informed interventions need to be built and adapted based on community voice for increased effectiveness and equity. The innovative work led by MDPH OPGS highlights why and how lived experience is critical to advancing equity within problem gambling research, programs, and services.

Acknowledgements We are grateful to all who contributed to the Ambassador Project, especially each Ambassador, Brian Robbins, and Efrain Baez, for their time, expertise, and dedication. We appreciate Lindsey Tucker, Associate Commissioner at the Massachusetts Department of Public Health, and Dr. Monica Bharel, Commissioner of the Massachusetts Department of Public Health, for their ongoing support and insight. Additionally, the communities who participated in planning processes and contributed their voices were integral to this work - this work would not be possible without them and we extend our deep gratitude.

Funding The Regional Planning Process was funded by the Public Health Trust Fund in Massachusetts.

Data availability Not applicable.

Code Availability Not applicable.

\section{Declarations}

Competing Interests The authors declare no competing interests..

Open Access This article is licensed under a Creative Commons Attribution 4.0 International License, which permits use, sharing, adaptation, distribution and reproduction in any medium or format, as long as you give appropriate credit to the original author(s) and the source, provide a link to the Creative Commons licence, and indicate if changes were made. The images or other third party material in this article are included in the article's Creative Commons licence, unless indicated otherwise in a credit line to the material. If material is not included in the article's Creative Commons licence and your intended use is not permitted by statutory regulation or exceeds the permitted use, you will need to obtain permission directly from the copyright holder. To view a copy of this licence, visit http://creativecommons.org/licenses/by/4.0/. 


\section{References}

Papers of particular interest, published recently, have been highlighted as:

- Of importance

-• Of major importance

1.• Alegría AA, Petry NM, Hasin DS, Liu S-M, Grant BF, Blanco C. Disordered gambling among racial and ethnic groups in the US: results from the National Epidemiologic Survey on Alcohol and Related Conditions. CNS Spectr. 2009;14(3):132-42 Available from: https://www.ncbi.nlm.nih.gov/pmc/articles/PMC2737691/ pdf/nihms125719.pdf Comprehensive study demonstrating racial inequities in problem gambling.

2.• Kessler RC, Hwang I, Labrie R, Petukhova M, Sampson NA, Winters KC, et al. DSM-IV pathological gambling in the National Comorbidity Survey Replication. Psychol Med. 2008;38(9):135160 Available from: https://www.ncbi.nlm.nih.gov/pmc/articles/ PMC2293303/pdf/nihms40981.pdf Important study showing comorbidities between problem gambling and substance use and mental health disorders.

3. Ahuja M, Cunningham-Williams R, Werner KB, Bucholz KK. Risk factors associated with gambling involvement among a national sample of African American and European American young adults. J Subst Abus Alcohol. 2018;6(3).

4. Ortiz V, Hernández H. Responsible gambling: public health and social justice considerations to inform research, policy, and practice. In: Responsible Gambling. Oxford University Press; 2019. p. $111-31$.

5. Miller HE, Thomas SL. The problem with 'responsible gambling': impact of government and industry discourses on feelings of felt and enacted stigma in people who experience problems with gambling. Addict Res Theory. 2018;26(2). Study integrating the voices of those with lived experience in gambling-related research.

6. Miller HE, Thomas SL, Robinson P. From problem people to addictive products: a qualitative study on rethinking gambling policy from the perspective of lived experience. Harm Reduct J. 2018;15(16).

7. Marshall Z, Dechman MK, Minichiello A, Alcock L, Harris GE. Peering into the literature: a systematic review of the roles of people who inject drugs in harm reduction initiatives. Drug Alcohol Depend. 2015;151:1-14. Recent systematic review examining peer-to-peer approaches and lived experience in harm reduction initiatives.

8. Watling D, Preece M, Hawgood J, Bloomfield S. Developing an intervention for suicide prevention: a rapid review of lived experience involvement. 2014;2020.

9. Werner KB, Cunningham-williams RM, Bucholz KK. Patterns of gambling and substance use initiation in African American and White adolescents and young adults. 2020;34(2):382-91.

10.• Korn DA, Shaffer HJ. Gambling and the health of the public: adopting a public health perspective. J Gambl Stud. 1999;15(4). Seminal piece applying a public health perspective to problem gambling.

11. Shaffer HJ, Korn DA. Gambling and related mental disorders: a public health analysis. Annu Rev Public Health. 2002;23:171-212.

12. Bronfenbrenner U. Ecological models of human development. In: International Encyclopedia of Education. 2nd ed. Oxford: Elsevier; 1994.

13. Messerlian C, Derevensky J, Gupta R. Youth gambling problems: a public health perspective. Health Promot Int. 2005;20(1):69-79.
14. Centers for Disease Control and Prevention. Social Determinants of health: know what affects health. 2020 [cited 2020 Nov 19]. Available from: https://www.cdc.gov/socialdeterminants/index. htm.

15. Magnan S. Social determinants of health 101 for health care five plus five. Natl Acad Med Perspect. 2017;1-9. Available from: https://nam.edu/social-determinants-of-health-101-for-health-carefive-plus-five/.

16. Massachusetts Department of Public Health, Massachusetts Gaming Commission. Strategic plan: services to mitigate the harms associated with gambling in Massachusetts. 2016. Available from: https://www.mass.gov/doc/strategic-plan-for-services-to-mitigatethe-harms-associated-with-gambling-in-massachusetts-0/ download.

17. Massachusetts Department of Public Health. Department of Public Health. 2020 [cited 2020 Nov 19]. Available from: https://www. mass.gov/orgs/department-of-public-health.

18. World Health Organization. Community engagement module B5 [PowerPoint slides].

19. Massachusetts Department of Public Health Office of Problem Gambling Services. Community Engagement Reports.

20. Petry NM. Gambling and substance use disorders: current status and future directions. Am J Addict. 2007;16(1):1-9 Available from: https://www.tandfonline.com/doi/abs/10.1080/ 10550490601077668.

21. Petry NM, Stinson FS, Grant BF. Comorbidity of DSM-IV pathological gambling and other psychiatric disorders: results from the National Epidemiologic Survey on Alcohol and Related Conditions. J Clin Psychiatry, Available from: https://www.ncbi. nlm.nih.gov/pubmed/15889941. 2005;66(5):564-74.

22. Bassuk EL, Hanson J, Greene RN, Richard M, Laudet A. Peerdelivered recovery support services for addictions in the United States: a systematic review. J Subst Abus Treat. 2016;63:1-9.

23. Ashford RD, Curtis B, Brown AM. Peer-delivered harm reduction and recovery support services: initial evaluation from a hybrid recovery community drop-in center and syringe exchange program. Harm Reduct J. 2018;15(1):52. Available from. https://doi.org/10. 1186/s12954-018-0258-2.

24. Latkin CA. Outreach in natural settings: the use of peer leaders for HIV prevention among injecting drug users' networks. Public Heal Rep [Internet]. 1998;113 Suppl 1(Suppl 1):151-9. Available from. https:/www.ncbi.nlm.nih.gov/pubmed/9722820.

25. Petry NM, Rash CJ, Alessi SM. A randomized controlled trial of brief interventions for problem gambling in substance abuse treatment patients. J Consult Clin Psychol. 2016;84(10):874-86.

26. Quilty LC, Wardell JD, Thiruchselvam T, Keough MT, Hendershot CS. Brief interventions for problem gambling: a meta-analysis. PLoS One. 2019;14(4):1-17 Available from: https://www.ncbi. nlm.nih.gov/pubmed/30995229.

27. Valente TW, Pumpuang P. Identifying opinion leaders to promote behavior change. Heal Educ Behav. 2007;34(6):881-96 Available from: https://www.ncbi.nlm.nih.gov/pubmed/17602096.

28. Eddie D, Hoffman L, Vilsaint C, Abry A, Bergman B, Hoeppner B, et al. Lived experience in new models of care for substance use disorder: a systematic review of peer recovery support services and recovery coaching. Front Psychol. 2019;10:1052 Available from: https://www.ncbi.nlm.nih.gov/pubmed/31263434.

29. Massachusetts Department of Public Health Office of Problem Gambling Services. Massachusetts Ambassador Project Impact Brief. 2019.

Publisher's Note Springer Nature remains neutral with regard to jurisdictional claims in published maps and institutional affiliations. 